\title{
Radiological progression and its predictive risk factors in silicosis
}

\author{
H S Lee, W H Phoon, T P Ng
}

\begin{abstract}
Objectives-To investigate the risk factors predicting radiological progression in silicosis in a prospective cohort study of patients with silicosis who were previously exposed to silica from granite dust.

Methods-From among a total of 260 patients with silicosis contracted from granite work, 141 with available serial chest $x$ ray films of acceptable quality taken over a period of 2 to 17 (mean 7.5) years, were selected for study. Ninety four $(66.7 \%)$ had ended exposure 5 or more years perviously (mean 10.1 years, maximum 28 years). Radiological progression was assessed by paired comparison of the initial and most recent radiographs, with two or more steps of increase in profusion of small opacities according to the 12 point scale of the International Labour Organisation (ILO) classification of radiographs of pneumoconiosis, taken from the majority reading by a panel of three independent readers.
\end{abstract}

Results-Overall, $37 \%$ of patients with silicosis had radiological evidence of progression. From the initial radiographs, 24 $(31.6 \%)$ of those with radiological profusion category $1,15(37.5 \%)$ of those with radiological profusion category 2 , and 13 $(52 \%)$ of those with complicated silicosis (including all seven with category 3 profusion of small opacities) showed radiological progression. As expected, progression was more likely to be found after longer periods of follow up (the interval between the two chest $x$ ray films) with a $20 \%$ increased odds of progression for every additional year of follow up. After adjustment for varying intervals of follow up, the probability of radiological progression was found to be significant if large opacities were present in the initial chest $x$ ray film. Progression was also less likely to be found among those who had ended exposure to silica longer ago, although the result was of borderline significance $(p=0.07)$. Tuberculosis was also associated with increased likelihood of progression (borderline significance).

Conclusions-There is a high probability of radiological progression in silicosis after high levels of exposure to granite dust among workers who were followed up for up to $\mathbf{1 7}$ years. A significant risk factor is the extent of radiological opacities in the initial chest $x$ ray film. The probability of progression is also likely to be reduced with longer periods after the end of exposure.

(Occup Environ Med 2001;58:467-471)

Keywords: silicosis; radiological progression; granite quarry

There are few longitudinal studies investigating the natural course and rate of radiological progression of silicosis and its predictive risk factors among workers exposed to silica dust. ${ }^{1-4}$ Previous investigators have followed up various occupational groups exposed to silica, which included sandblasters, ${ }^{1}$ slate workers, ${ }^{2}$ granite quarry workers, ${ }^{3}$ coal workers, ${ }^{4}$ and iron ore miners. ${ }^{5}$ They differ in the type, intensity, and duration of exposure to silica. The durations of follow up also differ, with the maximum durations of follow up ranging between less than 16 months (in slate pencil workers), and up to 20 years (in coal workers).

The predictive risk factors reported to be associated with radiological progression in silicosis included the duration ${ }^{12}$ and concentrations ${ }^{134}$ of past exposure to silica, initial profusion of radiological small opacities, ${ }^{1-3}$ simple and complicated disease, ${ }^{1-3}$ tuberculosis infection, ${ }^{5}$ age at initial film (lower probability of progression in older men), ${ }^{1}$ race (higher probabilities of progression in black than in white men), ${ }^{1}$ and the time interval between $x$ ray examination (higher probabilities of progression with higher time intervals). ${ }^{1}$

The objective of this study was to describe the radiological progression of silicosis among patients with silicosis who were previously exposed to silica from granite quarries and the risk factors that were associated with such progression.

Material and methods

Silicosis is a legally notifiable occupational disease since 1970 in Singapore, and annual chest $x$ ray films have been a legal requirement for all granite quarry workers since 1972. Doctors are required to notify cases of silicosis or suspected silicosis to the Ministry of Manpower. The diagnosis of silicosis is confirmed by doctors from the Department of Industrial Health Ministry of Manpower, on the basis of chest radiographs showing reticular-nodular shadowing of profusion grade of at least 1/1 (according to the International Labour Organisation (ILO) international standard classification of radiographs of pneumoconiosis), a definite history of occupational exposure to silica, and the exclusion of other causes of the radiological opacities. The Department of Industrial Health keeps a register of all confirmed cases of silicosis in Singapore. These 
Table 1 Respirable dust concentrationa ( $8 \mathrm{~h}$ time weighted average) of crusher workers at granite quarries in Singapore

\begin{tabular}{llll}
\hline Year & $n$ & $\begin{array}{l}\text { Mean } \\
\left(\mathrm{mg} / \mathrm{m}^{3}\right)\end{array}$ & $\begin{array}{l}\text { Samples } \\
\left(\%>1 \mathrm{mg} / \mathrm{m}^{3}\right)\end{array}$ \\
\hline 1973 & 116 & 3.07 & 85.3 \\
1976 & 141 & 1.27 & 42.5 \\
1977 & 296 & 1.24 & 48.6 \\
1978 & 291 & 1.12 & 50.1 \\
1985 & 270 & 1.26 & 55.2 \\
1986 & 217 & 0.99 & 34.6 \\
1987 & 114 & 0.83 & 25.4 \\
\hline
\end{tabular}

cases were followed up every 3 years with a chest $x$ ray film and a questionnaire. There were 260 cases of silicosis contracted from work in granite quarries (about $78 \%$ of all cases of silicosis in various industries and occupations). Of the 260 cases, 141 who had available serial chest $x$ ray films of acceptable quality were selected for study.

RADIOGRAPHIC READINGS

For each subject, the earliest chest radiograph at the time of initial diagnosis or thereafter and the most recent chest radiograph, both of acceptable quality, were selected. The $x$ ray films were read independently by three experienced readers according to the international standard classification of radiographs of pneumoconiosis 1980 . All the readers are specialists in occupational medicine with interest in respiratory disease. In the national follow up and medical surveillance programmes, they have been engaged for many years in the reading of chest $x$ ray films with the standard classification to assess compensation for workmen with pneumoconiosis. The two films for each subject were read as follows: $(a)$ the first (initial) film was read and categorised; $(b)$ the second (most recent) film was placed next to the first film and then read and categorised.

Progression was defined as increase of: two or more steps in the 12 point scale of radiological profusion of small opacities, or a one step in the size of small or large opacities, or the development of large opacities not previously present. For assessing the progression of the size of small opacities, the first letter of the pair (for example, $\mathrm{p}$ in the case of $\mathrm{p} / \mathrm{q}$ ) was evaluated. Progression was deemed to be present when at least two readers agreed on any one of the given criteria.
QUESTIONNAIRE INFORMATION

At the time of diagnosis, a detailed history of occupation, smoking, and medical treatment was taken for each subject. The occupational history included the nature and dates of employment of each job ever held in any quarries or other relevant workplaces in chronological order. A history of treatment for pulmonary tuberculosis was recorded for every subject. At each follow up visit, the information on occupation, smoking, and medical treatment was updated. There was no information on the use of respirators and this was therefore not taken into account in the analysis.

EXPOSURE TO DUST

All the subjects had confirmed silicosis and had previously worked in granite quarries. They were nearly all exposed to high airborne concentrations of silica dust either in and around the crusher plants (as mechanics, greasers, operators, labourers, or supervisors) or at the quarry face (drillers, stone breakers, or general labourers). The concentrations of dust in the exposure of crusher workers in various granite quarries in Singapore from 1973 to 1987 are shown in table 1. Concentrations of dust were higher before 1975, before the implementation of dust control measures.

STATISTICAL ANALYSIS

The logist procedure in the statistical analysis system (SAS) software package was used to perform logistic multiple regression for categorical response data (silicosis progression). This procedure was used to examine simultaneously the relation between radiological progression and several possible predictive factors, including age at initial chest radiography, initial radiological category, smoking, interval between initial and most recent chest radiograph, duration of exposure to silica dust, history of pulmonary tuberculosis, and the duration since exposure at the time of the most recent chest radiograph.

\section{Results}

STUDY POPULATION

The 141 subjects were confirmed to have silicosis between 1970 and 1990. The age at the time of the initial chest $x$ ray film ranged

Table 2 Characteristics of subjects with silicosis that progressed $v$ those with disease that had not progressed

\begin{tabular}{|c|c|c|c|c|}
\hline & $\begin{array}{l}\text { All subjects } \\
(n=141)\end{array}$ & $\begin{array}{l}\text { Disease progressed } \\
(n=52)\end{array}$ & $\begin{array}{l}\text { Disease not } \\
\text { progressed }(n=89)\end{array}$ & pValue \\
\hline Age at initial chest $x$ ray film (mean (SD)) & $53.7(8.5)$ & $51.7(8.8)$ & $54.9(8.2)$ & $\mathrm{p}=0.03$ \\
\hline Age at first exposure (mean (SD)) & $27.3(9.2)$ & $26.7(9.0)$ & $27.7(9.4)$ & NS \\
\hline Duration of exposure (mean (SD)) & $23.4(9.7)$ & $23.4(9.0)$ & $23.1(10.1)$ & NS \\
\hline \multicolumn{5}{|l|}{ Smoking $(\mathrm{n}(\%))$ : } \\
\hline Non-smoker & $23(16.3)$ & $10(19.2)$ & $13(14.6)$ & \multirow{3}{*}{ NS } \\
\hline Ex-smoker & $44(32.1)$ & $19(36.5)$ & $25(28.1)$ & \\
\hline Current smoker & $74(52.5)$ & $23(44.2)$ & $51(57.3)$ & \\
\hline \multicolumn{5}{|l|}{ Radiological category (initial chest $x$ ray film, $\mathrm{n}(\%)$ ): } \\
\hline Simple (category 1) & $76(53.9)$ & $24(46.2)$ & $52(58.4)$ & \multirow{3}{*}{ NS } \\
\hline Simple (category 2) & $40(28.4)$ & $15(28.9)$ & $25(28.1)$ & \\
\hline Complicated & $25(17.7)$ & $13(25.0)$ & $12(13.5)$ & \\
\hline Interval between chest $x$ ray films (initial and most recent, $\mathrm{y}$ ) & $7.5(3.5)$ & $8.6(3.8)$ & $6.9(3.2)$ & $\mathrm{p}=0.004$ \\
\hline $\begin{array}{l}\text { Subjects who had ended exposure at least } 1 \text { y before the most } \\
\text { recent chest } x \text { ray film }(\mathrm{n}(\%))\end{array}$ & $123(87.2)$ & $44(84.6)$ & $79(88.8)$ & NS \\
\hline $\begin{array}{l}\text { Time since exposure ended to most recent chest } x \text { ray film (y, } \\
\text { mean (SD)) }\end{array}$ & $8.8(6.5)$ & $8.2(5.9)$ & $9.2(7.0)$ & NS \\
\hline History of tuberculosis & $47(33.3)$ & $22(42.3)$ & $25(28.1)$ & NS \\
\hline
\end{tabular}

$t$ Test was done for continuous variables and $\chi^{2}$ test for categorical variables. 
from 32 to 80 (mean 53.7) years (table 2). The age at first exposure to silica ranged from 15 to 62 (mean 27.3) years. The duration of exposure to silica ranged from 4 to 50 (mean $23.4)$ years. Seventy four $(52.5 \%)$ were smokers, 44 (32.1) were ex-smokers, and only 23 $(16.3 \%)$ were non-smokers. Forty seven (33.3\%) had a history of pulmonary tuberculosis. All the subjects except one (who started exposure in 1976) had started exposure before 1975. Only five subjects started exposure in 1970 or later.

On the initial chest radiograph, 76 (53.9\%) had simple silicosis with radiological profusion category $1,40(28.4 \%)$ had simple silicosis with profusion category 2 , and seven subjects had category 3 profusion. Twenty five subjects $(17.7 \%)$ had evidence of large opacities (complicated silicosis). All the seven subjects who were classified as radiological category 3 also had large opacities. The interval between the most recent and initial chest radiograph ranged from 2 to 17 (mean 7.5) years. At the time of the most recent chest radiograph, $123(87.2 \%)$ had already ended exposure at least 1 year previously. Ninety four $(66.7 \%)$ had ended exposure 5 or more years ago. Among those who had ended exposure at least 1 year ago, the mean was 10.1 years and the maximum was 28 years.

RADIOLOGICAL PROGRESSION

Overall, $52(36.9 \%)$ had radiological evidence of progression. From the initial radiological state of the disease, $24(31.6 \%)$ of those with simple silicosis radiological profusion category $1,15(37.5 \%)$ of those with simple silicosis radiological profusion category 2 , and 13 $(52 \%)$ of those with complicated silicosis (including the seven with category 3 profusion of small opacities) showed evidence of radiological progression. Among those with simple silicosis, $36(31 \%)$ had an increase in profusion of small opacities, $18(15.5 \%)$ had an increase in size of small opacities, and $19(16.4 \%)$ had developed large opacities. Nine $(22.5 \%)$ who had simple silicosis (category 2), and 10 (13.2\%) who had simple silicosis (category 1 ) developed large opacities. However, the differences in development of large opacities between category 1 and 2 profusion were not significant $(p=0.3)$. Among those with complicated silicosis, 11 (44\%) had an increase in size of large opacities, five $(20 \%)$ had an increase in profusion of small opacities, and five $(20 \%)$ had an increase in size of small opacities.

The mean interval between chest $x$ ray films was 8.6 years for subjects with progression of disease and 6.9 years for those without $(\mathrm{p}=0.004$, table 2$)$. The age at initial chest radiography was 51.7 years for subjects with progression and 54.7 years for those without $(p=0.03)$. About $42 \%$ of those with progression had a history of tuberculosis compared with $28 \%$ among those without. However, this was not significant. Subjects without progression had been without exposure for longer (9.2 years) than those with progression (8.2 years) but this was not significant. There was also no significant difference in the age at first exposure, duration of exposure, smoking, and initial radiological category between subjects with progression and those without.

Multiple logistic regression analysis of risk factors showed that the probability of radiological progression was significantly determined by the presence of large opacities in the initial chest $x$ ray film $(p=0.01)$ and the interval between the two chest radiographs $(p=0.001$, table 3). Progression was also less likely to be found among those who had ended exposure to silica earlier $(p=0.07)$. There was no significant association with age at initial chest radiograph, exposure duration, history of tuberculosis, smoking, or period since the end of exposure. In a stepwise selection model (model 2) which included only the significant factors, those with category 3 profusion or large opacities on initial chest $x$ ray film were five times more likely to progress than those with simple silicosis category 1 , after adjustment for varying year of follow up $(p=0.005)$. Those with category 2 profusion were twice as likely to progress than those with category 1 , but this was not significant.

Table 3 Multiple logistic regression analysis of risk factors predicting radiological progression

\begin{tabular}{|c|c|c|c|c|}
\hline Risk factors & $\begin{array}{l}\text { Parameter } \\
\text { estimate }\end{array}$ & $S E$ & p Value & OR $(95 \% C I)$ \\
\hline \multicolumn{5}{|l|}{ Model 1 (saturated model with forced variables): } \\
\hline Age at initial chest $x$ ray film & -0.0202 & 0.028 & NS & $0.98(0.93$ to 1.03$)$ \\
\hline Interval between chest $x$ ray films (y) & 0.2390 & 0.071 & $\mathrm{p}=0.001$ & $1.27(1.10$ to 1.46$)$ \\
\hline \multicolumn{5}{|l|}{ Profusion category at initial chest $x$ ray film: } \\
\hline Category 1 & Reference & & & 1.00 \\
\hline Category 2 & 0.7080 & 0.464 & NS & $2.03(0.88$ to 4.70$)$ \\
\hline Category 3 or complicated ${ }^{\star}$ & 1.3987 & 0.569 & $\mathrm{p}=0.01$ & $4.05(1.34$ to 12.23$)$ \\
\hline Duration of exposure $(y)$ & -0.0100 & 0.026 & NS & $0.99(0.95$ to 1.05$)$ \\
\hline History of pulmonary tuberculosis & 0.6729 & 0.439 & NS & $1.96(0.83$ to 4.64$)$ \\
\hline Ever smoker & -0.4463 & 0.537 & NS & $0.64(0.22$ to 1.84$)$ \\
\hline Time since exposure ended to latest chest $x$ ray film (y) & -0.0726 & 0.037 & NS & $0.93(0.87$ to 1.01$)$ \\
\hline \multicolumn{5}{|l|}{ Model 2 (stepwise selection model with significant factors only): } \\
\hline Interval between chest $x$ ray films (y) & 0.1772 & 0.0572 & $\mathrm{p}=0.02$ & $1.19(1.07$ to 1.34$)$ \\
\hline \multicolumn{5}{|l|}{ Profusion category at initial chest $x$ ray film: } \\
\hline Category 1 & Reference & & & 1.00 \\
\hline Category 2 & 0.6374 & 0.4398 & NS & 1.89 (0.80 to 4.48$)$ \\
\hline Category 3 or complicated ${ }^{\star}$ & 1.608 & 0.566 & $\mathrm{p}=0.005$ & $4.99(1.65$ to 15.14$)$ \\
\hline
\end{tabular}

^All cases with category 3 profusion also had large opacitioes. 


\section{Discussion}

This study has shown that $37 \%$ of granite workers with silicosis showed radiological evidence of progression during a period of follow up that ranged from 2 to 17 years (mean 7.5 years). It should be noted that these were confirmed cases of silicosis arising from high levels of exposure to silica, and we are therefore not able to study initial progression from normal to category 1 .

For comparison, in a group of slate pencil workers, ${ }^{2}$ exposure to silica was very high, progression was extremely rapid, and the overall rate of radiological progression was $31.9 \%$ over a very short (16 month) follow up. Exceptionally high levels of exposure to silica and unusually rapid progression were also encountered in a group of 61 sandblasters with silicosis ${ }^{1}: 64 \%$ showed radiological progression over a follow up period of 1-7 years. The clinical disease investigated in these studies may be more aptly described as accelerated silicosis. The results of these studies therefore do not represent the usual conditions of exposure and natural course of disease progression encountered in chronic silicosis.

Among coalworkers who were exposed to unusual concentrations of respirable quartz, $38 \%$ of workers showed radiological progression of at least one profusion category on the 12 point scale over about a 20 year period and this was attributed to silicosis rather than coalworker's pneumonconiosis. ${ }^{4}$ Over a similar long period of follow up, radiological progression was found in $24 \%$ of workers with silicosis from a Swedish iron mine where exposures to respirable quartz were about $0.8 \mathrm{mg} / \mathrm{m}^{3}$ in the $1950 \mathrm{~s}$ and below $0.05 \mathrm{mg} / \mathrm{m}^{3}$ in the late $1970 \mathrm{~s}^{5}$ Another study of granite workers in Hong Kong ${ }^{4}$ followed up 73 workers with silicosis for a similar duration as in this study, 2-10 (mean 7.2) years, and found radiological progression in $48 \%$. These studies are more apt to describe the usual conditions of exposure and the natural history of disease commonly encountered in chronic silicosis, and are akin to those described in the present study.

It should also be noted, however, that all these studies differ not only in the intensity and duration of exposure to silica, clinical type of silicotic disease, and the durations of follow up, but the methods of determining radiological progression were also different. In this study we used the side by side method of presenting radiographs, with known sequence of $x$ ray films, for paired comparison. The side by side method has been recommended as the preferred method compared with the randomised independent method, one reason being its simplicity and practicality. ${ }^{6-8}$ Independent readings of the serial films are perhaps more likely to give unbiased readings of progression; however, it is also likely to give more variable readings, including regressive changes. As the readers knew the sequence of the films, there is the distinct possibility of a bias towards interpreting only positive progression, and disallowing possible regressive changes. We defined radiological progression as an increase of two or more steps in profusion of small opacities on the 12 point scale of the ILO classification of 1980 , as determined from the median readings of three independent readers. The study of slate pencil workers ${ }^{2}$ also used a minimum two steps increase in profusion of small opacities, but this was not determined from a panel of multiple readers. The study of coalworkers' silicosis ${ }^{4}$ used a minimum one step increase in profusion of small opacities from a independent panel reading by three readers. In the study of sandblaster's silicosis, ${ }^{1}$ no explicit criteria were used to determine radiological progression, although panel reading by three readers was also used. The granite worker's silicosis study in Hong Kong ${ }^{3}$ used the randomised independent method of presenting $x$ ray films for panel reading by three readers. These differences in methods of determining radiological progression may account for some degree of disparities in observed probabilities of progression of silicosis in different studies. It should be noted that the ILO 12 point scale for assessing profusion is not a linear one. A two step progression from a lower category may not be the same as a two step progression in a higher category. However, we have assumed a linear relation in our analysis.

We found that the probabilities of radiological progression in silicosis were higher in those with greater profusion of small opacities in simple silicosis, and in those with complicated disease (large opacities) at the initial chest radiograph. The probability was five times higher in this category than in those with simple silicosis category 1 . Radiological progression was found in $31.6 \%$ of those with category 1 radiological profusion of simple silicosis, $37.5 \%$ of those with category 2 radiological profusion, and $52 \%$ of those with complicated silicosis. This relation with radiological profusion of small opacities has been consistently reported in most studies. In silicosis in slate pencil workers, the probabilities of progression were $82.5 \%$ among those with complicated silicosis, $60 \%$ among those with simple silicosis of category 3 profusion, $26 \%$ among those with category 2 profusion, and $29.5 \%$ among those with category 1 profusion. In silicosis among sandblasters, $72 \%$ of those with complicated silicosis and $52 \%$ of those with simple silicosis showed radiological progression; differences in probabilities of progression by subcategories of profusion of small opacities were not reported. ${ }^{1}$ In the study of granite workers in Hong Kong, $55 \%$ of those with complicated silicosis and $45 \%$ of those with simple silicosis showed radiological progression. ${ }^{3}$ There is, therefore, uniformly clear evidence that the initial radiological severity of silicosis is a strong determining factor of future progression of disease.

We found that progression was more likely to be found with longer periods of follow up, with a $20 \%$ increased odds of progression for every additional year of follow up. This has not been investigated in other studies except in that of silicosis in sandblasters. ${ }^{1}$ In that study, it was also found that the longer the time between $x$ ray films, the more likely for progression to have occurred. The probabilities of progression were $79 \%$ in those in whom the serial films 
spanned more than 45 months and $52 \%$ in those in whom the films spanned less than 45 months.

There is thus strong supporting evidence that pulmonary nodular fibrosis in silicosis is progressive over time. Moreover, it would seem to be so, even when exposure to silica had stopped for as long as 28 years as in this study. However, there was some supporting evidence in this study that radiological progression might possibly be slowed with longer periods after the end of exposure. This has not been investigated previously. Although the results were of borderline significance, there was a $7 \%$ reduced likelihood of progression for every year that the subject had stopped exposure to silica before the time of the most recent chest $x$ ray film.

Smoking as a risk factor for rapid radiological progression in silicosis has been investigated in the studies of sandblasters ${ }^{1}$ and slate pencil workers. ${ }^{2}$ Neither of these studies nor the present study showed a strong determining effect of cigarette smoking on radiological progression. This may not be surprising as our study subjects were all confirmed to have silicosis based on the initial criterion of a profusion of $1 / 1$ or more. Smoking may be a significant factor in contributing to smaller opacities below a profusion of $1 / 1$. Age at initial film was not found to be significantly associated with progression in this study. However, in silicosis in sandblasters, ${ }^{1}$ older men were found to be less likely to progress than younger men, probably because of a survival effect. Tuberculosis as a risk factor for radiological progression was investigated in only one previous study ${ }^{4}$; in concurrence with the study of silicosis in Swedish iron-miners, we found tuberculosis to be also positively related to a greater likelihood of progression, although this was not significant.

The role of exposure to silica was not fully explored in the present study, as only duration of exposure was investigated as a surrogate variable. By contrast with previous studies which indicated that duration of exposure to silica was related to the likelihood of radiological progression, ${ }^{12}$ we were not able to show such an association with progression, independently of the extent of radiological opacities. The few studies that had data on dust measurement reported that the average silica concentration of exposure was also related to the likelihood of radiological progression. ${ }^{14}$ The independent effect of exposure to silica dust is difficult to show, because it is strongly related to the extent of radiological opacities in silicosis, and hence, to a great extent, one of the variables will substitute for the other in the analysis.

In conclusion, there is a high probability of radiological progression in silicosis from exposure to granite dust among workers who were followed up for up to 17 years. A significant risk factor is the extent of radiological opacities in the initial chest $x$ ray film. The probability of progression is also likely to be reduced with longer periods after the end of exposure. As expected, the risk of progression increases with the duration of interval between examinations.

1 Hughes JM, Jones RN, Gilson JC, et al. Determinants of progression in sandblasters' silicosis. Ann Occup Hyg 1982;26:701-12.

2 Saiyed HN, Chatterjee BB. Rapid progression of silicosis in slate pencil workers: II. A follow up study. Am f Ind Med

$3 \mathrm{Ng}$ TP, Chan SL, Lam KP. Radiological progression and lung function in silicosis: a ten year follow up study. $B M F$ 1987;295:164-8.

4 Miller BG, Hagen S, Love RG, et al. Risk of silicosis in coalworkers exposed to unusual concentrations of respirable quartz. Occup Environ Med 1998;55:52-8.

5 Jorgensen HS. Silicosis in the iron-ore mine in Kiruna, Sweden, and the future need for silicosis control. Int Arch Occup Environ Health 1986;58:251-7

6 Amandus HE, Reger RB, Pendergrass EP, et al. The pneumoconiosis: methods of measuring progression. Chest 1973;63:736-43.

7 Liddell FDK, Morgan WKC. Methods of assessing serial films of the pneumoconiosis: a review. I Soc Occup Med 1978;28:6-15.

8 Oldham PD. Assessing the radiological progression of simple pneumoconiosis. British fournal of Industrial Medicine 1968;25:150-2.

\section{Correspondence and editorials}

Occupational and Environmental Medicine welcomes correspondence relating to any of the material appearing in the journal. Results from preliminary or small scale studies may also be published in the correspondence column if this seems appropriate. Letters should be not more than 500 words in length and contain a minimum of references. Tables and figures should be kept to an absolute minimum. Letters are accepted on the understanding that they be subject to editorial revision and shortening.

The journal also publishes editorials which are normally specially commissioned. The Editor welcomes suggestions regarding suitable topics; those wishing to submit an editorial, however, should do so only after discussion with the Editor. 